\title{
Activation of sonic hedgehog signaling in oral squamous cell carcinomas: a preliminary study
}

\author{
Marcilei Eliza Cavicchioli Buim PhD ${ }^{a, *, 1}$, Clarissa Araújo S. Gurgel DDS, PhD ${ }^{b, 1}$, \\ Eduardo Antônio Gonçalves Ramos MD, PhD ${ }^{b}$, Silvia Vanessa Lourenço DDS, PhD ${ }^{c}$, \\ Fernando Augusto Soares MD, PhD ${ }^{\mathrm{a}, \mathrm{c}}$
}

\author{
${ }^{a}$ Department of Anatomical Pathology, Hospital A.C. Camargo, 01509-900 São Paulo, Brazil \\ ${ }^{\mathrm{b}}$ Laboratory of Pathology and Molecular Biology, Oswaldo Cruz Foundation, 05508-000 Bahia, Brazil \\ ${ }^{\mathrm{c}}$ Department of General Pathology, Dental School, University of São Paulo, 40296-710 Brazil
}

Received 6 September 2010; revised 12 December 2010; accepted 21 December 2010

\section{Keywords: \\ Sonic hedgehog; \\ Oral squamous cell \\ carcinoma; \\ Gene expression; \\ Immunohistochemistry}

\begin{abstract}
Summary Sonic hedgehog signaling is important for human development, and aberrant regulation of this pathway can result in the development of tumors. The aim of this study was to examine the expression of sonic hedgehog signaling molecules in oral squamous cell carcinoma. By quantitative real-time polymerase chain reaction, the expression of SHH, SMO, PTCH-1, and GLI-1 was analyzed in 30 oral squamous cell carcinoma cases and 8 samples of nonneoplastic oral mucosa and associated to clinical pathologic features. The expression of $\beta$-catenin, cyclin D1, Wnt-1, and Egfr was evaluated by immunohistochemistry in 26 available cases of oral squamous cell carcinoma. Normal oral mucosa from healthy individuals was negative for all genes that were evaluated. SHH, PTCH-1, SMO, and GLI-1 were not expressed in nonneoplastic oral mucosa, and low levels of GLI-1 were observed in nonneoplastic oral mucosa that was adjacent to the tumor. All oral squamous cell carcinoma cases expressed high levels of PTCH-1, SMO, and GLI-1 and were devoid of SHH. The expression of SMO was associated with clinical stage $(P=.022)$ and a borderline association in cervical lymph node metastasis $(P=.053) . P T C H-1$ expression showed a strong correlation with $S M O(r s=0.64 ; P<.001)$ and $G L-1(r s=0.70 ; P<.001) ; S M O$ and $G L I-1$ also correlated with each other $(r s, 0.55 ; P<.001)$. All proteins evaluated were expressed as cyclin D1 (92\% of samples), $\beta$-catenin (73\%), Egfr (46\%), or Wnt$1(32 \%)$. Our data demonstrate that sonic hedgehog signaling is activated in oral squamous cell carcinoma and suggest that this pathway mediates its tumorigenesis.
\end{abstract}

(C) 2011 Elsevier Inc. All rights reserved.

\section{Introduction}

ts This study was supported by grants 07/50609-0 and 98/14335-2 from Fundação de Amparo à Pesquisa do Estado de São Paulo, São Paulo, Brazil.

* Corresponding author. Department of Anatomical Pathology, 01509900 São Paulo, Brazil.

E-mail address: mebuim@hotmail.com (M. E. Cavicchioli Buim).

${ }^{1}$ First and second authors had the same contribution in this work.

Oral neoplasms constitute $5 \%$ of all human cancers, and oral squamous cell carcinoma (OSCC) accounts for more than $90 \%$ of diagnosed cases, ranking it as the sixth most frequent tumor globally [1].

Pathogenesis studies on OSCC are critical because despite the advances in its diagnosis and treatment, patient survival 
and quality of life remain unsatisfactory. Loss of speech, difficulties in chewing and swallowing, pain, facial deformities, and serious psychologic problems often develop [2].

Some groups have suggested that certain signaling pathways, such as sonic hedgehog ( $\mathrm{SHH}$ ) and wingless type (WNT) are associated with carcinogenesis and, consequently, mediate the initiation and progression of human malignant tumors $[3,4]$. The SHH pathway is one of the most important pathways in vertebrates, regulating many processes during embryonic development, including the formation of skin [5] and oral mucosa [6]. The sonic hedgehog protein (Shh) binds to the transmembrane receptor Patched, which liberates the transmembrane protein Smoothened (Smo) to initiate a series of intracellular cascades that effect the translocation of the transcription factor glioma-associated oncogene homolog 1 (Gli-1) into the nucleus [7,8], where it activates the transcription of genes that are related to cell proliferation and the cell cycle, such as GLI-1, PTCH-1 (Patched 1), transforming growth factor $\beta$ family members, CCND1, oncogene B-cell leukemia 2 (BCL2), WNT-1, epidermal growth factor receptor $(E G F R)$, and $\beta$-catenin $[9,10]$.

Activation of the SHH pathway, by loss of PTCH-1 function or mutational activation of SMO, occurs frequently in basocellular carcinomas and medulloblastomas [11]. Recently, dysregulated activation of SHH signaling has been observed in small cell lung cancer [12], pancreatic carcinoma [13], prostate cancer [14], and gastric cancer $[15,16]$.

Although many genetic alterations have been described in OSCC, little is known about the expression of genes that regulate SHH signaling in OSCC. Thus, the objectives of this study were to characterize the expression of genes in the SHH pathway (SHH, SMO, PTCH-1, and GLI-1) by quantitative real-time polymerase chain reaction (qPCR) and to identify any association between their expression and clinical pathologic features in patients with OSCC. Furthermore, we analyzed the expression of proteins whose transcription is activated by SHH signaling (cyclin D1, $\beta$-catenin, Egfr, and Wnt-1) by immunohistochemistry to increase our understanding of the pathogenesis of OSCC.

\section{Materials and methods}

\subsection{Patients}

This study, approved by the ethics committee of our institution (study no. 986/07), was performed using 30 cases of OSCC from excision biopsies, 5 cases of nonneoplastic oral mucosa that was adjacent to the primary tumor (NNT1), and 3 specimens of nonneoplastic oral mucosa (NNT2) that were harvested from healthy individuals who underwent extraction of third molars. These specimens were obtained from the Human Tumor Bank at A.C. Camargo Hospital in São Paulo, Brazil, between 2000 and 2006 and used to evaluate gene expression profiles by qPCR.
Of the 30 cases, 26 paraffin-embedded specimens were available for analysis by immunohistochemistry; paraffinembedded specimens from 4 cases were not suitable for such an analysis. Histologic grading was performed using parameters that were established by the World Health Organization (2005). Patients ranged in age from 30 to 90 years (mean age, 57 years). The clinical pathologic characteristics of the patients with OSCC are summarized in Table 1.

\subsection{Total RNA isolation and reverse transcription}

Total RNA was extracted from pulverized frozen tissue using Trizol (Invitrogen, Carlsbad, CA) according to the manufacturer's instructions. Genomic DNA was eliminated by Dnase I digestion (Invitrogen). The quality and purity of the RNA were assessed on an Agilent 2100 Bioanalyzer using the RNA 600 Nanolab Chip kit (Agilent Technologies, Waldbronn, GE). Total RNA was stored in RNAse-free distilled water at $-70^{\circ} \mathrm{C}$.

First-strand complementary DNA (cDNA) was synthesized from $1 \mu \mathrm{g}$ of total RNA using the High-Capacity cDNA Reverse-Transcription Kit (Applied Biosystems, Foster City, CA) in $20 \mu \mathrm{L}$ according to the manufacturer's instructions. The reactions were incubated at $25^{\circ} \mathrm{C}$ for 10 minutes, $37^{\circ} \mathrm{C}$ for 120 minutes, and $85^{\circ} \mathrm{C}$ for 5 seconds. The

Table 1 Clinical pathologic characteristics of patients with OSCC

\begin{tabular}{lll}
\hline Clinical pathologic parameters & Total (n) & $\%$ \\
\hline Sex & & \\
Male & 23 & 77 \\
Female & 07 & 23 \\
Size & 18 & \\
T1/T2 & 11 & 62 \\
T3/T4 & 01 & \\
Not available & & \\
Clinical stage & 12 & 40 \\
I and II & 18 & 60 \\
III and IV & & \\
Lymph nodes & 11 & 38 \\
N0 & 18 & 62 \\
N1-N3 & 01 & \\
No lymph node dissection & & \\
Perineural invasion & 15 & \\
No & 14 & \\
Yes & 01 & 37 \\
Not available & & \\
Vascular embolization & 20 & \\
No & 09 & \\
Yes & 01 & \\
Not available & 11 & \\
Histologic grade & & \\
Well differentiated & & \\
Poorly/moderately differentiated & 19 & \\
\hline
\end{tabular}


cDNA was stored at $-20^{\circ} \mathrm{C}$. Reverse-transcriptase efficiency was determined by amplification of $\beta$-actin gene.

\subsection{Quantitative real-time PCR (qPCR)}

Thirty OSCC samples, 5 samples of NNT1 (pooled into 1 group), and 3 samples of NNT2 (also pooled) were evaluated by qPCR. Primers for $S H H, S M O, P T C H-1, G L I-1$, and the control reference gene GAPDH were designed using PrimerExpress, version 3.0 (Applied Biosystems), based on sequences in GenBank (National Center for Biotechnology Information, www.ncbi.nlm.nih.gov/sites/entrez). Three endogenous genes ( $\beta$-actin, GAPDH, and HPRT1) were tested in all samples as normalizers. Based on the amplification curves using GeNorm [17], GAPDH was the most stable gene for normalization of the reactions. Table 2 shows the primer pairs that were used for qPCR.

A relative standard curve was constructed for all primers using a serial dilution of a small cell lung cancer line (H146) $(100,50,25,12.5$, and $6 \mathrm{ng} / \mu \mathrm{L})$. The standard curves of the target and reference genes were similar with regard to efficacy $(>90 \%)$.

PCR reactions were performed in duplicate on an ABI Prism 7900 (Applied Biosystems) in $20 \mu \mathrm{L}$, containing $10 \mathrm{ng}$ of the cDNA sample, $0.2 \mu \mathrm{mol} / \mathrm{L}$ of each primer, and $1 \mathrm{X}$ Syber-Green PCR Master Mix (Applied Biosystems). The amplification program consisted of 1 cycle of $50^{\circ} \mathrm{C}$ for 2 minutes and $95^{\circ} \mathrm{C}$ for 10 minutes, followed by 40 cycles of $95^{\circ} \mathrm{C}$ for 15 seconds and $60^{\circ} \mathrm{C}$ for 1 minute. To verify the specificity of the amplification, we performed a melting curve analysis, subjecting the samples to an initial denaturation at $95^{\circ} \mathrm{C}$ for 15 seconds, followed by incubation for 15 seconds at $60^{\circ} \mathrm{C}$ and a ramping step of $95^{\circ} \mathrm{C}$ at $0.1^{\circ} \mathrm{C}$ per second, with continuous detection of fluorescence.

Relative quantification was expressed as the ratio between the mean value of the target gene and the mean value of the reference gene $(G A P D H)$ in each sample. cDNA from a small lung cancer cell line (H146) was used to calibrate the reactions. The relative amount of PCR product that was generated from each primer set was

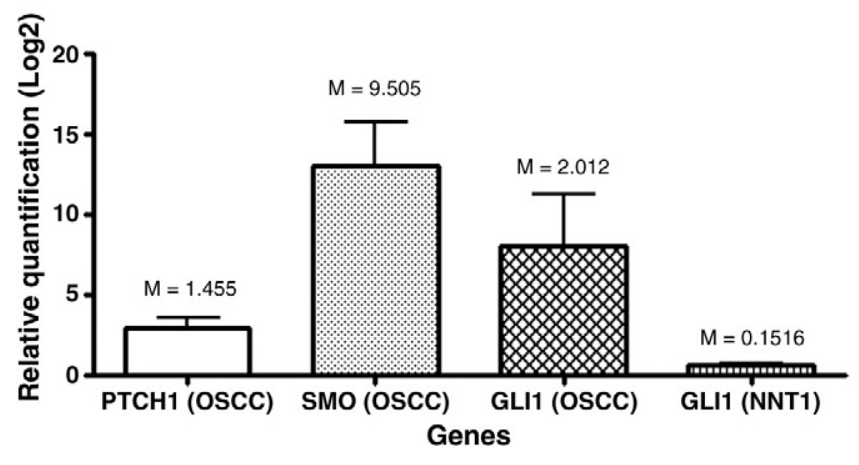

Fig. 1 Relative expression of PTCH-1, SMO, and GLI-1 in OSCC and NNT1. M indicates median.

determined based on the cycle threshold $(\mathrm{Ct})$ value, and relative quantification was calculated using a mathematical model, as described by Pfaffl [18].

\subsection{Immunohistochemistry}

To examine the expression of proteins in the $\mathrm{SHH}$ pathway, 4- $\mu \mathrm{m}$ sections were deparaffinized and rehydrated. Antigens were retrieved by boiling the sections in sodium citrate solution $(\mathrm{pH}, 6.0)$ in a pressure cooker for 15 minutes.

Slides were placed in 3\% hydrogen peroxide 3 times for 5 minutes each. The sections were blocked with Protein Block Serum-Free (Dako, Carpinteria, CA) at room temperature for 20 minutes.

The sections were incubated at room temperature for 2 hours with the following primary antibodies: rabbit monoclonal anti-cyclin D1 (prediluted; clone RBT-14; Bio SB, Inc, Santa Barbara, CA), rabbit monoclonal anti- $\beta$-catenin (1:100; clone 9582; Cell Signaling, Danver, MA), rabbit polyclonal anti-Wnt-1 (1:300; clone H89; Santa Cruz Biotechnology, Inc, Santa Cruz, CA), and monoclonal mouse anti-Egfr (1:500; clone H11; Dako, Carpinteria, CA). After being washed in phosphate-buffered solution 3 times for 5 minutes each, the sections were incubated using the indirect dextran polymer detection system (Novocastra Laboratories Ltd, Newcastle, UK) for 1 hour.

Table 2 Primer pairs, symbol, and accession number of SHH pathway genes for qPCR

\begin{tabular}{|c|c|c|c|}
\hline Identification & Symbol & Accession no. & Sequences $5^{\prime}$ à $3^{\prime}$ \\
\hline Sonic hedgehog homolog & $\mathrm{SHH}$ & NM_000193.2 & $\begin{array}{l}\text { F: GCGCCAGCGGAAGGTAT } \\
\text { R: CCGGTGTTTTCTTCATCCTTAAA }\end{array}$ \\
\hline Patched homolog 1 & PTCH1 & NM_000264.3 & $\begin{array}{l}\text { F: GGGTGGCACAGTCAAGAACAG } \\
\text { R: CGTACATTTGCTTGGGAGTCATT }\end{array}$ \\
\hline Smoothened homolog & $S M O$ & NM_005631.3 & $\begin{array}{l}\text { F: GGTTTGTGGTCCTCACCTATGC } \\
\text { R: GGAGGTCTTGCCCGAGAGA }\end{array}$ \\
\hline Glioma-associated oncogene homolog 1 & $G L I 1$ & NM_005269.1 & $\begin{array}{l}\text { F: CGCTGCGAAAACATGTCAAG } \\
\text { R: CCACGGTGCCGTTTGGT }\end{array}$ \\
\hline Glyceraldehyde-3-phosphate dehydrogenase & $G A P D H$ & NM_002046 & $\begin{array}{l}\text { F: CCAGGTGGTCTCCTCTGACTTC } \\
\text { R: GTGGTCGTTGAGGGCAATG }\end{array}$ \\
\hline
\end{tabular}

Abbreviations: F, forward; R, reverse.

Source: http://www.ncbi.nlm.nih.gov/sites/entrez. 
Table 3 Association between the expression of SMO, PTCH-1, and GLI-1 and the clinical pathologic parameters of patients with OSCC

\begin{tabular}{|c|c|c|c|c|c|c|c|c|c|c|}
\hline \multirow[t]{2}{*}{ Parameters } & \multirow[t]{2}{*}{ Total } & \multicolumn{3}{|l|}{ SMO } & \multicolumn{3}{|l|}{$\mathrm{PTCH}$} & \multicolumn{3}{|l|}{$G L I$} \\
\hline & & Mean & SD & $P$ & Mean & SD & $P$ & Mean & SD & $P$ \\
\hline \multicolumn{11}{|l|}{ Sex } \\
\hline Female & 07 & 13.88 & 6.7 & \multirow[t]{2}{*}{.135} & 2.98 & 1.21 & \multirow[t]{2}{*}{.178} & 1.29 & 0.51 & \multirow[t]{2}{*}{.540} \\
\hline Male & 23 & 10.49 & 2.0 & & 2.10 & 0.51 & & 7.84 & 4.83 & \\
\hline \multicolumn{11}{|l|}{ Size } \\
\hline $\mathrm{T} 1 / \mathrm{T} 2$ & 18 & 11.79 & 2.94 & \multirow[t]{2}{*}{.928} & 2.29 & 0.78 & \multirow[t]{2}{*}{.753} & 4.32 & 2.12 & \multirow[t]{2}{*}{.590} \\
\hline T3/T4 & 11 & 10.21 & 2.59 & & 2.18 & 0.54 & & 9.39 & 8.12 & \\
\hline \multicolumn{11}{|l|}{ Clinical stage } \\
\hline I and II & 12 & 18.47 & 3.70 & \multirow[t]{2}{*}{.022} & 3.55 & 1.36 & \multirow[t]{2}{*}{.236} & 6.94 & 4.02 & \multirow[t]{2}{*}{.611} \\
\hline III and IV & 18 & 8.51 & 1.89 & & 1.80 & 0.39 & & 6.83 & 5.4 & \\
\hline \multicolumn{11}{|l|}{ Vascular embolization } \\
\hline No & 20 & 11.31 & 2.58 & \multirow[t]{2}{*}{.706} & 2.37 & 0.65 & \multirow[t]{2}{*}{.925} & 3.49 & 1.80 & \multirow[t]{2}{*}{.572} \\
\hline Yes & 09 & 10.54 & 3.02 & & 2.04 & 0.65 & & 11.91 & 10.09 & \\
\hline \multicolumn{11}{|l|}{ Perineural invasion } \\
\hline No & 15 & 14.00 & 2.58 & \multirow[t]{2}{*}{.163} & 2.56 & 0.79 & \multirow[t]{2}{*}{.663} & 4.45 & 2.09 & \multirow[t]{2}{*}{.896} \\
\hline Yes & 14 & 8.00 & 2.62 & & 1.91 & 0.49 & & 9.26 & 8.14 & \\
\hline \multicolumn{11}{|l|}{ Histologic grade } \\
\hline Well differentiated & 11 & 10.09 & 3.41 & \multirow[t]{2}{*}{.525} & 2.34 & 0.59 & \multirow[t]{2}{*}{.397} & 2.26 & 0.55 & \multirow[t]{2}{*}{.899} \\
\hline Moderated differentiated & 19 & 11.49 & 2.39 & & 2.18 & 0.66 & & 9.33 & 6.32 & \\
\hline \multicolumn{11}{|l|}{ Lymph nodes } \\
\hline N0 & 11 & 18.47 & 3.70 & \multirow[t]{2}{*}{.053} & 3.55 & 1.36 & \multirow[t]{2}{*}{.559} & 6.93 & 4.02 & .323 \\
\hline N1-N3 & 18 & 8.51 & 1.89 & & 1.80 & 0.39 & & 6.83 & 5.41 & \\
\hline
\end{tabular}

The sections were incubated in 3,3'-diaminobenzidine tetrachloride (Dako, Carpinteria, CA). The slides were then lightly counterstained with hematoxylin, dehydrated in absolute ethanol and xylene, mounted in permanent mounting medium with cover slips, and examined under an optical light microscope.

Quantitative analysis was performed using ACIS III, version 3.1 (Dako, San Juan Capistrano, CA), which determines the percentage of cells whose nuclei are stained and measures the intensity of cytoplasmic and membranous staining. $\beta$-Catenin, Egfr, and Wnt-1 were considered to be down-regulated at staining intensities up to the mean value; at intensities above the mean value, OSCC cases were considered to be positive.
For cyclin D1, down-regulated cases were defined as those that harbored up to $20 \%$ stained nuclei. Positive cases were defined as those in which more than $20 \%$ of the nuclei were cyclin D1-positive.

\subsection{Statistical analysis}

The associations between gene expression and clinical pathologic parameters were determined by Mann-Whitney $U$ test. The correlation between genes (SMO, PTCH-1, and $G L I-1)$ was analyzed by Spearman test.

All $P$ values were based on 2 -tailed statistical analysis, and $P<.05$ was considered to be statistically significant. The

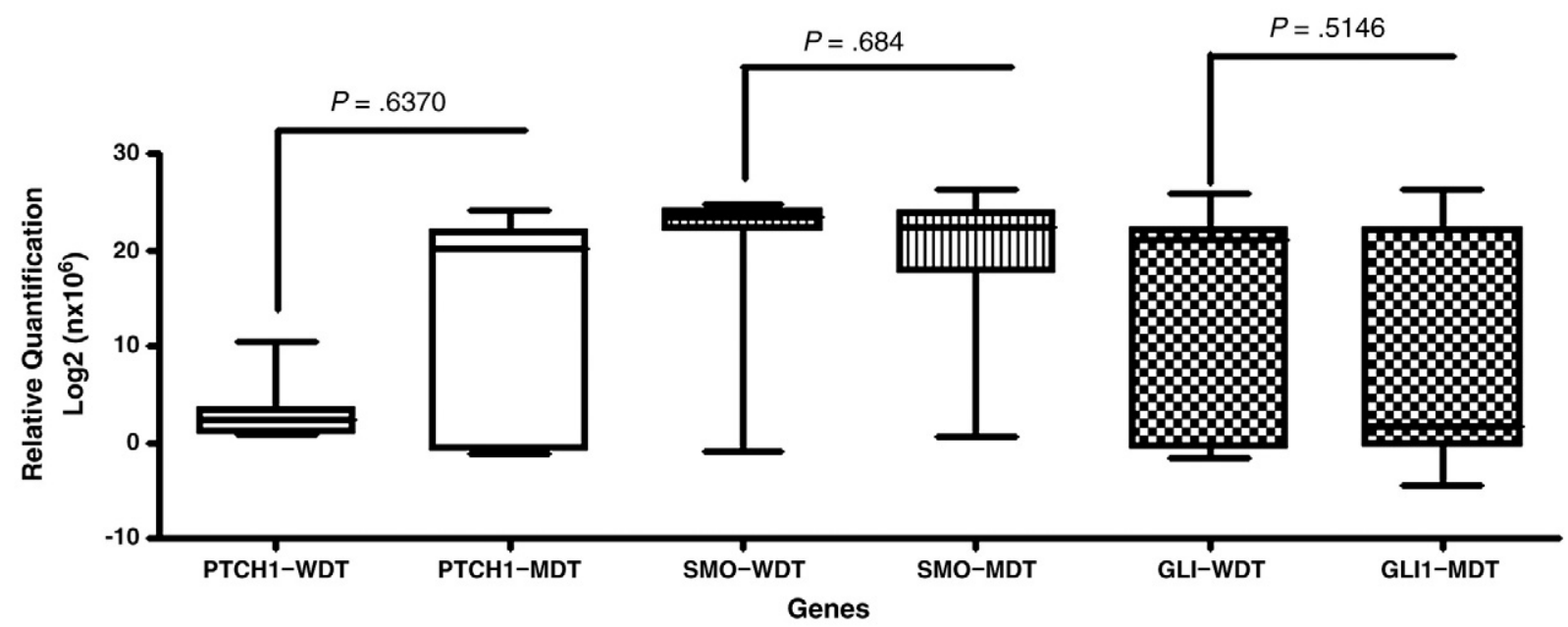

Fig. 2 Relative expression of PTCH-1,SMO, and GLI-1 between well-differentiated and moderately/poorly differentiated tumors. 
statistical analyses were performed using SPSS 17.0 (SPSS Inc, Chicago, IL).

\section{Results}

To investigate the expression profile of genes in the $\mathrm{SHH}$ signaling cascade in OSCC, we measured the expression of SHH, SMO, PTCH-1, and GLI-1 in 30 tumor samples and compared these results with clinical pathologic parameters. We also analyzed the expression of proteins of genes whose transcription is activated by SHH signaling (cyclin D1, $\beta$-catenin, Wnt-1, and Egfr) in 26 available samples.

$S H H, P T C H-1$, and $S M O$ were not expressed in either pool of normal oral tissue (NNT1 or NNT2). Normal oral mucosa from healthy individuals was negative for $G L I-1$ gene, but we observed low GLI-1 expression in the NNT1 group.

In all OSCC specimens, we noted high levels of $P T C H-1$, $S M O$, and GLI-1 and the absence of SHH. Fig. 1 shows the mean expression levels of these genes.

A robust and statistically significant correlation was found between the expression of PTCH-1 and SMO ( $r S=$ $0.688 ; P<.001)$, PTCH-1 and GLI-1 ( $r s=0.725 ; P<$ .001 ), and SMO and GLI-1 ( $r s=0.596 ; P=.001)$.

No gene was associated with sex, perineural invasion, histologic grade, or tumor size. However, SMO expression was linked to clinical stage $(P=.022)$, and a borderline association was observed in cervical lymph node metastasis $(P=.053$; Table 3$)$.

In moderately/poorly differentiated tumors, higher transcription levels of PTCH-1 were observed and compared with well-differentiated tumors $\left(P=.397^{\prime}\right.$ Fig. 2$)$, although this difference was not statistically significant. The expression of SMO and GLI-1 was similar between welldifferentiated tumors and moderately or poorly differentiated tumors (Fig. 2).

Twenty-four (92\%) of 26 OSCC cases were positive for cyclin D1. Nineteen $(73 \%)$ of 26 cases were $\beta$-catenin positive, 12 (46\%) of 26 cases expressed Egfr, and 8 (32\%) of 26 cases were positive for Wnt-1. Fig. 3 shows representative immunohistochemical stains of these molecules in OSCC samples.

\section{Discussion}

In human and animal models, activation of the $\mathrm{SHH}$ pathway is associated with the development of tumors through diverse mechanisms [19]. For example, in medulloblastoma and basal cell carcinomas, SHH signaling can initiate because of PTCH-1 mutations [11], whereas in small cell lung cancer [12] and intestinal adenocarcinoma [15], its activation is associated with high expression of the SHH ligand.

Gene expression in the SHH pathway in OSCC has not been well studied. Only 3 reports have examined this area;
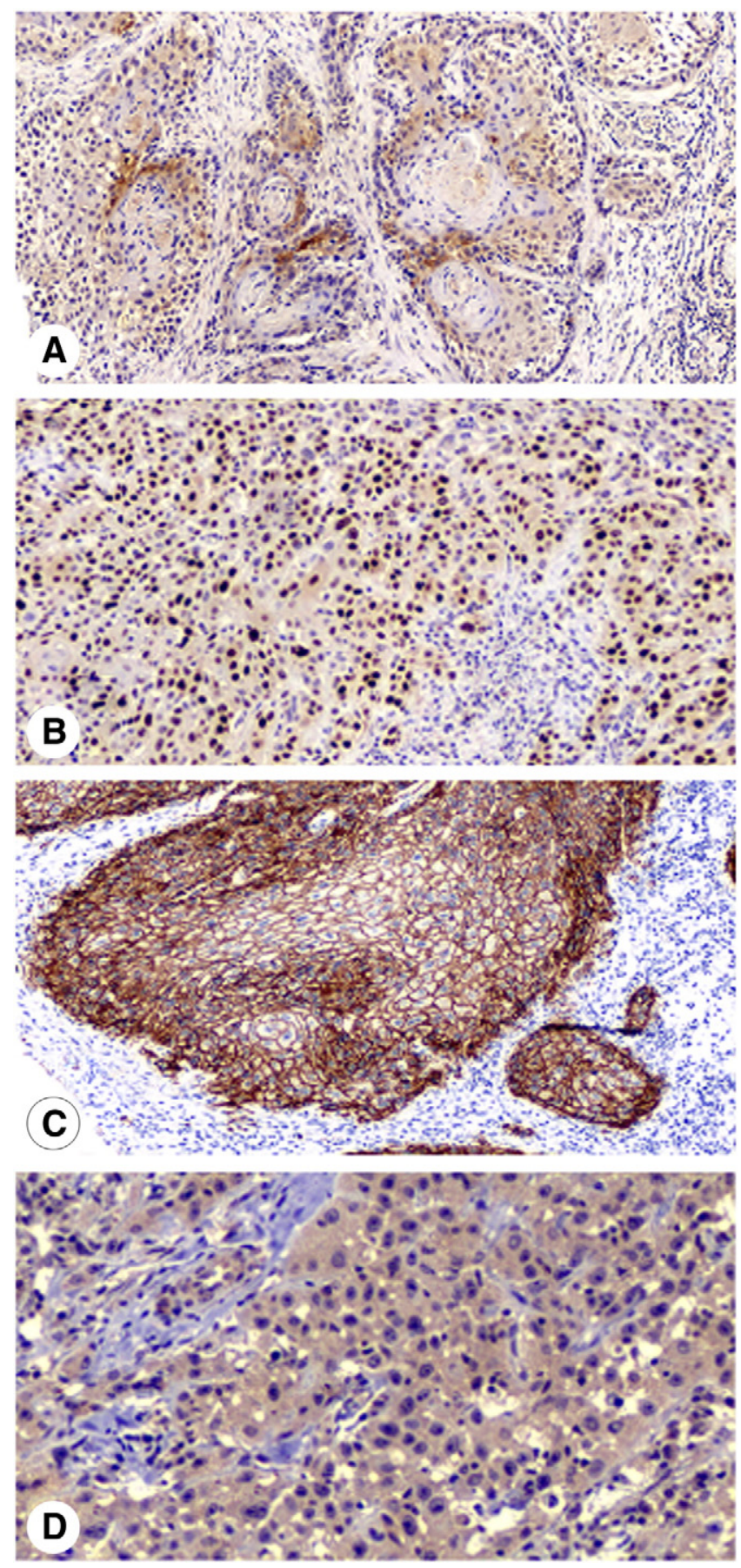

Fig. 3 Immunohistochemistry of OSCC samples: $\beta$-catenin (A), cyclin D1 (B), Egfr (C), and Wnt-1 (D).

one study used OSCC cell lines [20], and another used an experimental Xenopus laevis model [21]. Schneider et al [22] assessed the expression of SHH signaling proteins in head and neck squamous cell carcinomas. Here, we found the transcriptional levels of genes in the SHH pathway in human OSCC.

We did not observe SHH, PTCH-1, SMO, or GLI-1 transcripts in normal oral mucosa in healthy individuals, suggesting that the SHH pathway is inactive in these tissues. 
Schneider et al [22] failed to note any expression of SHH signaling proteins in oral normal mucosa. However, in our study, low levels of GLI-1 were expressed in NNT1.

Our results are consistent with findings from other studies, which have reported that many pathways that are essential for embryonic development, such as $\mathrm{SHH}$, are quiescent in adult tissues [22]. When these pathways are activated aberrantly in adult tissues, they are frequently oncogenic $[4,5,22]$. In an experimental X laevis model, Dahman et al [21] observed that the development of squamous cell carcinoma and basal cell carcinoma depended on the amounts of injected GLI-1 messenger RNA. Furthermore, they described the presence of GLI-1 transcripts in normal basal cells in tissue that was adjacent to the primary tumor. Considering these data, our findings on GLI-1 suggest that cells of the nonneoplastic oral mucosa next to the tumor comprise tumor clones that were undetected histologically.

All OSCC specimens expressed high levels of PTCH-1, SMO, and GLI-1 and no SHH. Nishimaki et al [20] observed that 5 of 14 OSCC cell lines expressed $S H H$ transcripts. In Schneider et al [22], 30 of 53 samples studied were weakly or moderately positive for SHH protein. Although the ligand Shh constitutes the basic mechanism by which the canonical SHH pathway is activated in embryonic tissues, in tumors, such activation might be effected by disparate mechanisms. In neoplasms that are associated with Gorlin syndrome, in which this pathway has been characterized most thoroughly, SHH does not appear to participate; moreover, abnormalities in PTCH-1, SMO, and GLI-1 have been demonstrated in cancer $[4,23]$.

Although the tumor suppressor gene PTCH-1 is a negative regulator of the SHH pathway, its expression in OSCC implicates that SHH signaling is active because PTCH-1 is also a target of the transcription factor GLI-1 [23-25]. Similarly, GLI-1 is expressed in all tumors in which SHH signaling participates $[19,22]$ and indicates aberrant activation of this pathway $[22,26]$. Our results suggest that the SHH pathway is activated in OSCC and mediates its pathogenesis. The expression of proteins in SHH signaling [22] in OSCC and of other proteins in our study (cyclin D1, $\beta$-catenin, Wnt-1, and Egfr) confirms this model.

Correlations were observed between PTCH-1,SMO, and GLI-1 and between SMO and GLI-1 expressions. Although the mechanisms that regulate expression of SHH signaling genes are not well understood in cancers, GLI-1 and PTCH1 levels seem to increase and are accompanied by robust activation of the pathway by $S M O$ [26]. Xie et al [24] observed that GLI-1 messenger RNA levels increased in $S M O$-overexpressing embryonic fibroblasts; thus, neoplastic cellular transformation might be favored by increased GLI-1 activity in tumor stem cells.

Furthermore, we noted that OSCCs that had poor/ moderate differentiation had higher transcriptional levels of PTCH-1 than did well-differentiated tumors, although this difference was not statistically significant. In gliomas and soft tissue and bone sarcomas, high levels of GLI-1 are associated with more aggressive tumors [27]. In our study, $S M O$ transcription was linked to clinical stage and marginally associated with the presence of cervical lymph nodes. The relationship between molecular phenomena and clinical parameters should be examined more extensively in a larger number of cases.

Our results suggest that the SHH pathway is activated in OSCC. Although the functional mechanism of this pathway remains unknown, SHH signaling might constitute a central mechanism of OSCC tumorigenesis.

\section{Acknowledgments}

We would like to thank Anthony Escobedo for reviewing and editing this manuscript.

\section{References}

[1] Johnson N, Franceschi S, Ferlay J, et al. Squamous cell carcinoma. World Health Organization classification of tumors. Head and neck tumors; 2005. p. 168-73.

[2] Méndéz E, Cheng C, Farwell DG, et al. Transcriptional expression profiles of oral squamous cell carcinoma. Cancer 2002;95:1482-94.

[3] Reya T, Morrison SJ, Clarke MF, Weissman IL. Stem cells, cancer, and cancer stem cells. Nature 2001;414:105-11.

[4] Altaba AR, Sánchez P, Dahmane N. Gli and hedgehog in cancer: tumours, embryos and stem cells. Nat Rev Cancer 2002;2:361-72.

[5] Athar M, Tang X, Lee JL, et al. Hedgehog signalling in skin development and cancer. Exp Dermatol 2006;15:667-77.

[6] Cobourne MT, Sharpe PT. Expression and regulation of hedgehoginteracting protein during early tooth development. Connect Tissue Res 2002;43:143-7.

[7] Ingham PW, Mcmahon AP. Hedgehog signaling in anima development: paradigms and principles. Genes Dev 2001;15:3059-87.

[8] Taipale J, Beachy PA. The hedgehog and wnt signaling pathways in cancer. Nature 2001;411:349-54.

[9] Chari NS, McDonnell TJ. The sonic hedgehog signaling network in development and neoplasia. Adv Anat Pathol 2007;14:344-52.

[10] Katoh Y, Katoh M. Hedgehog target genes: mechanisms of carcinogenesis induced by aberrant hedgehog signaling activation. Curr Mol Med 2009;9:873-86.

[11] Hahn H, Christiansen J, Wicking C, et al. A mammalian patched homolog is expressed in target tissues of sonic hedgehog and maps to a region associated with developmental abnormalities. J Biol Chem 1996;271:12125-8.

[12] Watkins DN, Berman DM, Burkholder SG, et al. Hedgehog signalling within airway epithelial progenitors and in small-cell lung cancer. Nature 2003;422:313-7.

[13] Thayer SP, Magliano MP, Heiser PW, et al. Hedgehog is an early and late mediator of pancreatic cancer tumorigenesis. Nature 2003;425:851-6.

[14] Antón-Aparicio LM, García Campelo R, Cassinello Espinosa J, et al. Prostate cancer and Hedgehog signalling pathway. Clin Transl Oncol 2007;9:4220-8.

[15] Berman DM, Karhadkar SS, Maitra A, et al. Widespread requirement for Hedgehog ligand stimulation in growth of digestive tract tumours. Nature 2003;425:846-51.

[16] Morton JP, Mongeau ME, Klimstra DS, et al. Sonic hedgehog acts at multiple stages during pancreatic tumorigenesis. Proc Natl Acad Sci U S A 2007;104:5103-8. 
[17] Vandesompele J, Preter K, Pattyn F, et al. Accurate normalization of real-time quantitative RT-PCR data by geometric averaging of multiple internal control genes. Gen Biol 2002;3:34.1-34.11.

[18] Pfaffl MW. A new mathematical model for relative quantification in real-time RT-PCR. Nucleic Acids Res 2001;29:45.

[19] Hahn H, Wojnowsi L, Miller G, Zimmer A. The patched signaling pathway in tumorigenesis and development: lessons from animals models. J Mol Med 1999;77:459-68.

[20] Nishimaki H, Kasai K, Kozaki K, et al. A role of activated sonic hedgehog signalling for the cellular proliferation of oral squamous cell carcinoma cell lines. Biochem Biophys Res Commun 2004;314: 313-29.

[21] Dahman N, Lee J, Robins P, et al. Activation of the transcription factor GLI-1 and the sonic hedgehog signalling pathway in skin tumours. Nature 1997;389:876-81.
[22] Schneider S, Thurnher D, Kloimstein P, et al. Expression of the sonic hedgehog pathway in squamous cell carcinoma of the skin and the mucosa of the head and neck. Head Neck 2011;33:244-50.

[23] High A, Zedan W. Basal cell nevus syndrome. Curr Opin Oncol 2005; $17: 160-6$

[24] Xie J, Murone M, Luoh SM, et al. Activating Smoothened mutations in sporadic basal-cell carcinoma. Nature 1998;391:90-2.

[25] Wetmore C. Sonic hedgehog in normal and neoplastic proliferation: insight gained from human tumors and animal models. Curr Opin Genet Dev 2003;13:34-42

[26] Kasper M, Regl G, Frischauf AM, Aberger F. Gli transcription factor: mediators of oncogenic hedgehog signaling. Eur J Cancer 2006;4:437-45.

[27] Becher OJ, Hambardzumyan D, Fomchenko EI, et al. Gli activity correlates with tumor grade in platelet-derived growth factor-induced. Cancer Res 2008;668:2241-9. 